DOI: https://doi.org/10.15688/jvolsu3.2018.3.12

UDC 62-405.(045)

LBC 65.28

\title{
ANALYSIS OF THE WORLD EXPERIENCE IN DOMESTIC WASTE MANAGEMENT ${ }^{1}$
}

\author{
Alla E. Kalinina \\ Volgograd State University, Volgograd, Russian Federation \\ Anna S. Barakova \\ Volgograd State University, Volgograd, Russian Federation
}

\begin{abstract}
Comparative analysis of the world's best practices in the management of solid domestic waste has shown that in the EU and the US a careful sorting of the waste and extracting a useful component, which is later introduced into production cycles, has been carried out for quite a long time. The authors note that in European countries the greatest amount of waste comes from industry and in particular from mining and construction. The EU is implementing a number of projects in the field of waste processing and recycling, which have begun to increase the use of aluminum waste, processing of which leads to the production of high-quality alloys. In Russia, the secondary use of aluminum is extremely poorly developed, and most of the waste that is processed in the EU is to be disposed of in Russia. Taking into account the energy resources necessary for the production of metal from primary raw materials, the prospects for the development of the processing industry become evident. It has also been revealed that Russian energy-related manufacturing enterprises do not implement recycling technology, which is so widely used in Europe and the US, i.e. the resulting waste after treatment is put back into production and additional sources of energy are obtained at the outlet, which could be useful in various branches of the economy. The proposed prospects for the effective organization of industrial waste processing are the expansion of the raw material base and the increase in the level of recycling, through the involvement of waste in production cycles.
\end{abstract}

Key words: ecology, solid domestic wastes, waste treatment, recycling, environment.

Citation. Kalinina A.E., Barakova A.S. Analysis of the World Experience in the Organization of Domestic Waste Management Systems. Vestnik Volgogradskogo gosudarstvennogo universiteta. Seriya 3, Ekonomika. Ekologiya [Science Journal of Volgograd State University. Global Economic System], 2018, vol. 20, no. 3, $\stackrel{\infty}{\circ}$

ن УДК 62-405.(045)

ББК 65.28

\section{АНАЛИЗ МИРОВОГО ОПЫТА ОБРАЩЕНИЯ С БЫТОВЫМИ ОТХОДАМИ ${ }^{1}$}

\author{
Алла Эдуардовна Калинина
}

Волгоградский государственный университет, г. Волгоград, Российская Федерация

Анна Сергеевна Баракова

Волгоградский государственный университет, г. Волгоград, Российская Федерация 
Аннотация. Компаративный анализ передового мирового опыта в сфере обращения с твердыми бытовыми отходами показал, что в странах ЕС и США уже достаточно долгое время осуществляется тщательная сортировка отходов и извлечение полезного компонента, который позже внедряется в производственные циклы. Авторы отмечают, что в европейских странах наибольшее количество отходов поступает из промышленности, в частности из горнодобывающей промышленности и строительства. В странах ЕС реализуется ряд проектов в области переработки и утилизации отходов, положивших начало увеличению использования отходов алюминия, переработка которых приводит к получению сплавов высокого качества. В России вторичное использование алюминия развито крайне слабо, большая часть отходов, которые перерабатываются в странах ЕС, в РФ подлежат захоронению. Принимая во внимание энергетические ресурсы, необходимые для производства металла из первичного сырья, становятся очевидны перспективы развития перерабатывающей отрасли. Также выявлено, что российские производственные предприятия, связанные с энергетикой, не реализуют технологию рециклинга, широко применяющуюся в Европе и США, то есть образовавшиеся отходы после обработки запускают в производство, получают дополнительные источники энергии, которые могут использоваться в различных отраслях хозяйства. Предлагаемые перспективы для эффективной организации переработки промышленных отходов - это расширение сырьевой базы и повышение уровня рециклинга за счет вовлечения отходов в производственные циклы.

Ключевые слова: экология, твердые бытовые отходы, переработка отходов, рециклинг, окружающая среда.

Цитирование. Калинина А. Э., Баракова А. С. Анализ мирового опыта обращения с бытовыми отходами // Вестник Волгоградского государственного университета. Серия 3, Экономика. Экология. - 2018. - Т. 20 , № 3. - C. 120-125. - DOI: https://doi.org/10.15688/jvolsu3.2018.3.12

Образование отходов загрязняет окружающую среду и создает экономическое напряжение на земле. Отходы приводят к ненужной потере природных ресурсов, таких как вода и энергия. Истощения природных ресурсов можно избежать, только уменьшив потребление или грамотно использовав отходы, основываясь на восстановлении ресурса, а также избегая необязательного выделения отходов и потребления энергии. В современных условиях человечество стремится достигнуть нулевых показателей отходов, то есть создать безотходные предприятия.

Вопросы переработки отходов во вторичное сырье становятся все важнее для мировой экономики. На сегодняшний день активно проектируются и строятся предприятия замкнутых циклов движения отходов и безотходные производства, которые позволяют извлекать из сырья все необходимые полезные составляющие. Хотя в течение последних 30 лет вблизи крупных промышленных предприятий и городов происходило складирование всевозможных отходов производства и потребления, что негативно сказывается на состоянии окружающей среды и здоровье людей.

В Австралии, например, региональные правительства уже осознали важность максимального сокращения твердых бытовых отходов (ТБО), о чем свидетельствует тот факт, что в Южной Австралии было создано правительственное отделение Zero Waste SA, ориентирующееся на выработку мер по сокращению отходов [3, p. 2-4].

В Европе, по последним подсчетам, каждый год образуется порядка 3000 млн т различного рода отходов. Это соответствует 3,8 т отходов на душу населения в Западной Европе, 4,4 т в странах Центральной и Восточной Европы и 6,3 т в странах Восточной Европы, Кавказа и Центральной Азии [4, p. 119]. Общее количество отходов в большинстве стран Западной и Восточной Европы, Кавказа и Центральной Азии (по которым имеются данные) продолжает расти. По Центральной и Восточной Европе картина более разнородная: в некоторых странах (Чехия, Венгрия и Польша) количество отходов увеличилось, однако снизилось в других (Эстония и Словакия).

По данным рисунка видно, что в европейских странах наибольшее количество отходов поступает из промышленности, а в частности из горнодобывающей промышленности и строительства. В первую очередь это обусловлено высоким развитием данных отраслей, а также их значимостью для жизни населения. Однако существенно сократить отходы, поступающие из этой сферы деятельности, невозможно на данном этапе, так как 


\section{Экология}

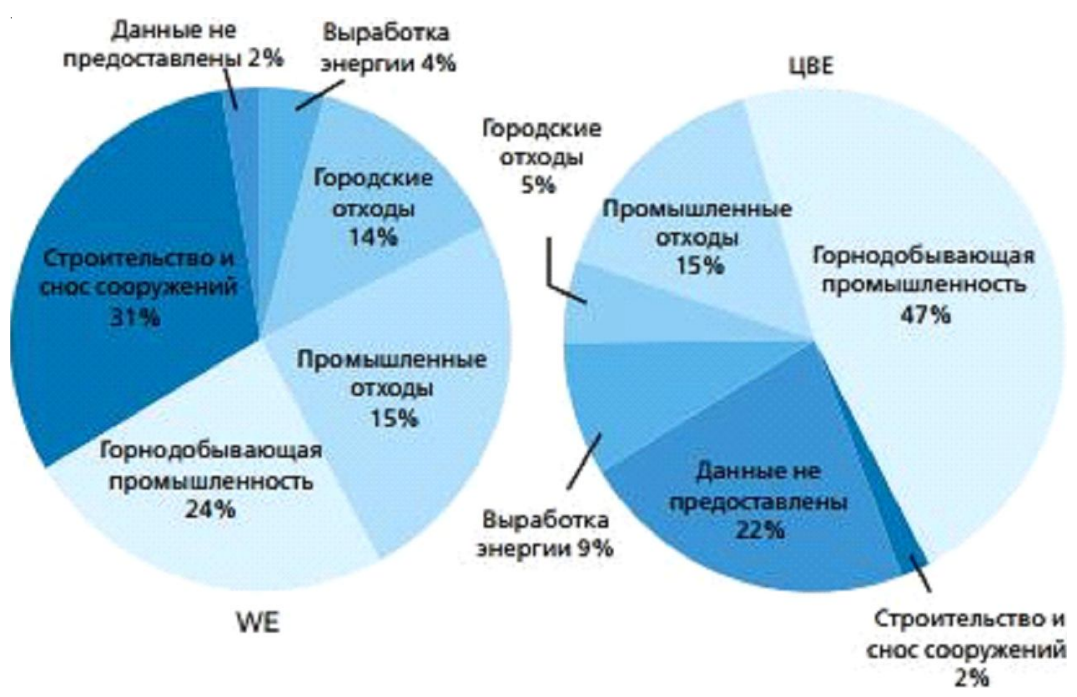

Рисунок. Общее количество образующихся отходов

в Западной Европе и Центральной и Восточной Европе по секторам экономики

Примечание. Составлено авторами по: [4, с. 190].

страны Европы не смогут сократить свою промышленность в силу недостатка природных ресурсов и высокой плотности населения.

Уровень переработки отходов в европейских странах различен. Больше всего отходов (от $30 \%$ до $50 \%$ и более) подвергаются вторичной переработке в Швеции, Дании, Швейцарии, Германии, Бельгии, Норвегии, Австрии и Голландии. Меньше всего (до $15 \%$ ) перерабатывают Великобритания, Исландия, Португалия и Греция. Для сравнения: в России на сегодняшний день перерабатывается порядка 2 \% отходов [1].

Российская и зарубежная практика в вопросах использования отходов как вторичного ресурса находится на разных уровнях. В странах ЕС и США уже достаточно долгое время осуществляется тщательная сортировка отходов и извлечение полезного компонента, который позже внедряется в производственные циклы. К тому же в случае вторичного использования сырья основное количество затрат вкладывается на первоначальном этапе, при закупке оборудования и обучении квалифицированного персонала, а далее начинает поступать материальная выгода в виде полезного компонента, зачастую не уступающего добываемым материалам. Особенно это важно для небольших государств с малым количествам ресурсов.

Рассмотрим ситуацию в области обращения с отходами в Венгрии. В 2000-2012 гг. в стране был переходный период в экономике, что вызвало ряд споров и конфликтов, в том числе и в области обращения с отходами, в частности касаемо свалок и захоронения.

По данным ЕС, наибольшие затраты венгерского государственного бюджета в области охраны окружающей среды были направлены именно на создание новых видов утилизации отходов и внедрение вторичного использование сырья. На тот момент большинство технологий по захоронению отходов устарело, и, хоть и количество отходов по сравнению с предыдущими годами существенно уменьшилось, опасность для окружающей среды возрастала.

Благодаря софинансированию ЕС значительное количество устаревших технологий было заменено на новые. К тому же количество образования отходов продолжало сокращаться, что позитивно сказывалось на состоянии окружающей среды.

Что касается ТБО, можно сделать вывод, что в Венгрии в основном по-прежнему используется захоронение отходов (80\%), сжиганию подвергается около $6 \%$, остальное переработано или скомпостировано.

Стоит отметить, что основные конфликты, связанные с обращением с отходами, возникают на почве финансового дефицита, обусловленного высоким уровнем безработицы в стране. 
В Венгрии нет как такового активного стремления к переходу на безотходное производство или увеличение вторичного использования сырья [2, р. 117]. Это связано с тем, что, во-первых, количество образовывающихся на территории страны отходов сокращается, во-вторых, с вполне приемлемой экологической ситуацией в стране, что обусловлено, как нам кажется, малым количеством производств, а также грамотным и своевременным обращением с отходами, несмотря на все экономические и политические перестройки.

В Европейском союзе реализуется ряд проектов в области переработки и утилизации отходов. Один из таких проектов - RecycAl.

Задача проекта RecycAl - разработать инновационную технологию переработки низкосортного алюминиевого лома с высоким содержанием примесей в новое высококачественное сырье, пригодное для создания чистых алюминиевых сплавов [5].

Проект RecycAl стремится решать проблему роста межметаллического формирования $\mathrm{Fe}, \mathrm{Sn}$ и примеси $\mathrm{Mn}$ в переработанных сплавах алюминия посредством развития и демонстрации метода High Shear Processing (HSP), который может позволить отходам легкой степени тяжести быть преобразованными в сплав высокого качества, следовательно, увеличив использование отходов Al в ЕС.

Технология RecycAl основана на применении процессов отбора и плавления отдельных элементов сплавов, а также работе с жидкими металлами.

Метод High Shear Processing (HSP), включает чрезвычайно быстрый (скорость вращения $>10,000 \mathrm{c}^{-1}$ ) раскол цельного металла благодаря роторному миксеру, что позволяет превращать в жидкий металл отобранный материал. В этом процессе не возникает никаких проблем, ибо жидкий металл имеет вязкость, подобную воде, и обеспечивает очень низкое сопротивление ротору даже на высокой скорости.

Благодаря этому проекту и развитию новых технологий положено начало увеличению использования отходов алюминия, переработка которых приводит к получению сплавов высокого качества. В результате образованные в ЕС отходы алюминия будут оставаться там, тем самым значительно уменьшится экспорт. Благодаря этому будут сохраняться некото- рые ресурсы ЕС, которых и без того немного, а также значительно уменьшится загрязнение окружающей среды, в частности, выделение $\mathrm{CO}_{2}$. Это вызвано тем, что производство первичного алюминия требует большой затраты энергии и интенсивного выделения углерода. Поэтому следует довериться очищенным, переработанным алюминиевым отходам, которые можно использовать повторно, сохраняя их высокое качество.

Статистические данные показывают, что 1 т переработанного алюминия экономит:

- 1370 кг бокситных остатков;

- 9800 кг $\mathrm{CO}_{2}$, выделенных в процессе производства;

- 64 кг $\mathrm{SO}_{2}[5]$.

RecycAl будет сосредоточиваться на использовании смешанных алюминиевых отходов, в настоящее время накапливающихся в городских ТБО. Метод НSP преобразует все твердые отходы в сырье, которое может быть повторно использовано для производства алюминиевых сплавов высокого качества.

Проект реализуется во всех странах ЕС, и у него есть ряд партнеров, помогающих в ходе его осуществления, таких как «Suldouro», «Idalsa» и многие другие.

В 2016 г. было реализовано несколько мероприятий по осуществлению проекта, одним из которых является определение состава и качества получаемого материала.

Партнеры проекта «Suldouro» собрали две формы отходов: 1) содержащие использованные банки из-под напитков; 2) выброшенные металлы, содержащие алюминий. Отходы были сортированы «Suldouro», чтобы удалить неметаллические и пластмассовые части, и были посланы в «Idalsa» для переработки. «Idalsa» сделали слитки из каждого типа отходов, которые уже могут быть использованы как вторичное сырье и имеют высокое качество, о чем свидетельствует состав каждого переработанного слитка (см. таблицу).

Теперь команда «Brunel» делает вычисления, основанные на диаграмме строения алюминия, чтобы найти оптимальные условия обработки для переработанных составов, а также определить, сколько железа может быть удалено из каждого сплава [5]. Следующий шаг должен будет состоять в использовании HSP-сплавов в производстве. 
Состав материалов, полученных при переработке отходов, \%

\begin{tabular}{|l|c|c|c|c|c|c|c|c|}
\hline \multicolumn{1}{|c|}{ Тип отходов } & $\mathrm{Al}$ & $\mathrm{Fe}$ & $\mathrm{Si}$ & $\mathrm{Mg}$ & $\mathrm{Mn}$ & $\mathrm{Cu}$ & $\mathrm{Cr}$ & $\mathrm{Ti}$ \\
\hline $\begin{array}{l}\text { Выброшенные металлы, } \\
\text { содержащие алюминий }\end{array}$ & 96,83 & 0,884 & 0,934 & 0,010 & 1,115 & 0,134 & 0,016 & 0,015 \\
\hline $\begin{array}{l}\text { Использованные банки } \\
\text { из-под напитков }\end{array}$ & 95,77 & 1,058 & 1,377 & 0,007 & 1,025 & 0,435 & 0,054 & 0,024 \\
\hline
\end{tabular}

Примечание. Составлено авторами по: [5].

В России подобного рода проектов нет, а данный проект не реализуется, так как страна не входит в Евросоюз. Что касается обращения с алюминием в нашей стране, то его вторичное использование развито крайне слабо, большая часть отходов, которые перерабатываются в ЕС, у нас подлежат захоронению.

По данным статистики, емкость российского рынка алюминиевых банок оценивается приблизительно в 2-3 млрд штук в год. Учитывая вес банки даже небольшого объема, составляющий порядка 15 г, получаем количество затраченного алюминия, которое приблизительно составляет 30-40 тыс. т чистого металла. А если принять во внимание и энергетические ресурсы, необходимые для производства металла из первичного сырья, становятся очевидны перспективы развития перерабатывающей отрасли [6, p. 215].

В заключение стоит отметить, что российские производственные предприятия, связанные с энергетикой, не реализуют технологию рециклинга, так широко применяющуюся в Европе и США (то есть получившиеся отходы после обработки запускают обратно в производство и на выходе получают дополнительные источники энергии, которые могли бы пригодиться в различных отраслях хозяйства).

Однако это далеко не все возможности использования вторичного сырья. Например, черная и цветная промышленность при более тщательной обработке отходов может получать качественные металлические соединения, которые, в свою очередь, можно использовать при строительстве, а также внедрять в производство автотранспорта. Подобные способы переработки широко применяются в европейских странах в условиях острой нехватки ресурсов, что позволяет сэкономить финансовые затраты на добычу и уменьшает воздействие на окружающую среду.
Таким образом, российская и зарубежная практика в вопросах использования отходов как вторичного ресурса находится на разных уровнях. В странах ЕС и США уже достаточно долгое время осуществляется тщательная сортировка отходов и извлечение полезного компонента, позже внедряющегося в производственные циклы. К тому же в случае вторичного использования сырья основное количество затрат происходит на первоначальном этапе, при закупке оборудования и обучении квалифицированного персонала, а далее начинает поступать материальная выгода в виде полезного компонента, зачастую не уступающего добываемым материалам, что особенно важно для небольших государств с малым количествам ресурсов. Следовательно, перспективы для эффективной организации переработки промышленных отходов - это расширение сырьевой базы и повышение уровня рециклинга (степени замкнутости) за счет вовлечения отходов в производственные циклы.

\section{ПРИМЕЧАНИЕ}

1 Исследование выполнено при финансовой поддержке РФФИ, проект № 17-12-34008 «Организация эффективной региональной системы обращения с отходами и ее инфраструктуры: институциональный, экономический и правовой аспект».

\section{СПИСОК ЛИТЕРАТУРЫ}

1. Статья для «продвинутых»: международный опыт и ситуация в России в области обращения с отходами (обо всем понемногу) // Moscow Business School. - Электрон. текстовые дан. - Peжим доступа: https://mbschool.ru/articles/54980 (дата обращения: 05.04.2018). - Загл. с экрана.

2. Kumar, E. S. Integrated Waste Management / E. S. Kumar. - Croatia : InTech, 2011. - 548 p. 
3. Li, R. Y. M. Construction Safety and Waste Management: An Economic Analysis / R. Y. M. Li. Cham (Switzerland) : Springer International Publishing, 2015. - $137 \mathrm{p}$.

4. Pacheco-Torgal, F. Handbook of recycled concrete and demolition waste / F. Pacheco-Torgal, J. de Brito, J. Labrincha. - Australia : University of Western Sydney, 2013. -646 p.

5. Recycled Material Processed Ready for High Shear Process / RecycAl. - Electronic data. - Mode of access: http://www.recycal-project.eu/news/recycledmaterial-processed-ready-for-high-shear-process/ (date of access: 16.03.2018). - Title from screen.

6. Wang, L. K. Advances in Hazardous Industrial Waste Treatment / L. K. Wang, N. K. Shammas, Y. T. Hung. - Florida : CRC Press : Taylor \& Francis Group, 2009. - 544 p.

\section{REFERENCES}

1. Statia dlia «prodvinutykh»: mezhdunarodnyi opyt i situatsiia v Rossii v oblasti obrashcheniia s otkhodami (obo vsem ponemnogu) [Article for "advanced": international experience and Russian realities in the sphere of recycling (a little of everything)]. Moscow Business School. URL: https://mbschool.ru/articles/54980 (accessed 5 April 2018).

2. Kumar E.S. Integrated Waste Management. Croatia, InTech, 2011. 548 p.

3. Li R.Y.M. Construction Safety and Waste Management: An Economic Analysis. Switzerland, Springer International Publishing, 2015, pp. 2-4.

4. Pacheco-Torgal F., De Brito J., Labrincha J. Handbook of recycled concrete and demolition waste. Australia, University of Western Sydney, 2013. 646 p.

5. Recycled Material Processed Ready for High Shear Process. RecycAl. URL: http://www.recycalproject.eu/news/recycled-material-processed-readyfor-high-shear-process/ (accessed 16 March 2018).

6. Wang L.K., Shammas N.K., Hung Y.T. Advances in Hazardous Industrial Waste Treatment. Florida, CRC Press, Taylor \& Francis Group, 2009. $544 \mathrm{p}$.

\section{Information about the Authors}

Alla E. Kalinina, Doctor of Sciences (Economics), Professor, Department of Applied Informatics and Mathematical Methods in Economics, Volgograd State University, Prosp. Universitetsky, 100, 400062 Volgograd, Russian Federation, allakalinina@volsu.ru.

Anna S. Barakova, Student, Institute of Economics and Finance, Volgograd State University, Prosp. Universitetsky, 100, 400062 Volgograd, Russian Federation, barakova.anya@yandex.ru.

\section{Информация об авторах}

Алла Эдуардовна Калинина, доктор экономических наук, профессор кафедры прикладной информатики и математических методов в экономике, Волгоградский государственный университет, просп. Университетский, 100, 400062 г. Волгоград, Российская Федерация, allakalinina@volsu.ru.

Анна Сергеевна Баракова, студент института экономики и финансов, Волгоградский государственный университет, просп. Университетский, 100, 400062 г. Волгоград, Российская Федерация, barakova.anya@yandex.ru. 